\title{
ON THE NON-LINEAR DISTORTION OF WAVES GENERATED BY FLAT PLATES UNDER HARMONIC EXCITATIONS
}

Non-linear waves generated in an acoustic medium by flat plates under harmonic excitations were investigated first by Ginsberg [1] using the method of renormalization. However, since the method was applied to the velocity potential rather than to physical primitive quantities such as, for example, the velocity perturbations, the results obtained were not uniformly valid $[2,3]$. Uniformly valid solutions were derived independently by Nayfeh and Kelly [4] and Ginsberg [5]. Re-examining the results obtained, Ginsberg [6] showed that the solution for the complete wave field can be split into two parts, each of which is very similar to the solution for an outgoing simple wave. Nevertheless, essential differences seemed to remain which were interpreted as being due to a mutual interaction of both wave families.

It is the aim of the present communication to show that by introducing a different set of co-ordinates, which arises naturally from application of the analytical method of characteristics (see, e.g., references $[7,8]$ ), the complete solution can indeed be obtained as the superposition of two simple wave solutions and no mutual interaction terms exist.

For simplicity the notation adopted by Ginsberg [5] is used in what follows. Thus $t / \Omega$ and $L x, L z$ denote the physical time and Cartesian co-ordinates parallel and perpendicular to an infinite flat plate located at $z=0$. The half space $z>0$ contains an ideal gas of constant specific heats. Expressing the transverse displacement of the plate in the form

$$
w=\varepsilon L \cos (t) \sin (N \pi x)+O\left(\varepsilon^{2}\right),
$$

where $\varepsilon \ll 1$ characterizes the small amplitude of the vibration and assuming expansions for the velocity disturbances and the speed of sound (non-dimensionalized by $L \Omega$ ) of the form

$$
v_{x}=\varepsilon v_{1 x}+O\left(\varepsilon^{2}\right), \quad v_{z}=\varepsilon v_{1 z}+O\left(\varepsilon^{2}\right), \quad c=c_{0}+\varepsilon c_{1}+O\left(\varepsilon^{2}\right),
$$

one obtains, as the first order results,

$$
\begin{gathered}
v_{1 x}=-(N \pi / k) \cos (t-k z) \cos (N \pi x), \\
v_{1 z}=-\sin (t-k z) \sin (N \pi x), \\
c_{1}=-\frac{1}{2}(\gamma-1)(f / k) \sin (t-k x) \sin (N \pi x) .
\end{gathered}
$$

The parameter $k$ is related to the speed of sound in the unperturbed medium and the reduced frequency $f$ through the relationship

$$
k=\left(f^{2}-N^{2} \pi^{2}\right)^{1 / 2}, \quad f=1 / c_{0} .
$$

However, the expressions for $\vec{v}_{1}=\left(v_{1 x}, v_{1 z}\right)$ and $c_{1}$ can also be cast into the form

$$
\vec{v}_{1}=\vec{v}_{1}^{(1)}+\vec{v}_{1}^{(2)}, \quad c_{1}=c_{1}^{(1)}+c_{1}^{(2)},
$$

where

$$
\begin{array}{ll}
\vec{v}_{1}^{(1)}=-\frac{1}{2}(f / k) \vec{n}^{(1)} \cos [t-f(x \sin \theta+z \cos \theta)], & c_{1}^{(1)}=\frac{1}{2}(\gamma-1) \vec{v}_{1}^{(1)} \cdot \vec{n}^{(1)}, \\
\vec{v}_{1}^{(2)}=\frac{1}{2}(f / k) \vec{n}^{(2)} \cos [t-f(-x \sin \theta+z \cos \theta)], & c_{1}^{(2)}=\frac{1}{2}(\gamma-1) \vec{v}_{1}^{(2)} \cdot \vec{n}^{(2)}
\end{array}
$$


describe the propagation of two planar simple waves in the directions characterized by the unit normal vectors

$$
\vec{n}^{(1)}=(\sin \theta, \cos \theta), \quad \vec{n}^{(2)}=(-\sin \theta, \cos \theta), \quad \theta=\tan ^{-1}(N \pi / k) .
$$

In order to render the first order solution of the straightforward expansion (2) uniformly valid the analytical method of characteristics will be applied. To this end $x$ and $z$ are expanded in the forms

$$
\begin{aligned}
& x=x_{0}+\varepsilon x_{1}(\xi, \eta, t)+O\left(\varepsilon^{2}\right), \\
& z=z_{0}+\varepsilon z_{1}(\xi, \eta, t)+O\left(\varepsilon^{2}\right),
\end{aligned}
$$

where $\xi=$ const. and $\eta=$ const. are the characteristic surfaces

$$
\xi=t-f\left(x_{0} \sin \theta+z_{0} \cos \theta\right), \quad \eta=t-f\left(-x_{0} \sin \theta+z_{0} \cos \theta\right),
$$

associated with the two families (6) and (7) of outgoing waves. Insertion of the expansions (9) into the exact slope conditions for characteristic surfaces (see, e.g., reference [9]) yields, to first order,

$$
\begin{array}{r}
x_{1} \sin \theta+z_{1} \cos \theta=\int_{t_{a}}^{t}\left(\vec{v}_{1} \cdot \vec{n}^{(1)}+c_{1}\right) \mathrm{dt}, \\
-x_{1} \sin \theta+z_{1} \cos \theta=\int_{t_{a}}^{t}\left(\vec{v}_{1} \cdot \vec{n}^{(2)}+c_{1}\right) \mathrm{d} t,
\end{array}
$$

where the integration has to be carried out along bicharacteristics which generate the characteristic surfaces $\xi=$ const. and $\eta=$ const., respectively,

$$
\begin{array}{ll}
\xi=\text { const: } & \mathrm{d} x_{0}: \mathrm{d} z_{0}: \mathrm{d} t=\sin \theta: \cos \theta: f, \\
\eta=\text { const: } & \mathrm{d} x_{0}: \mathrm{d} z_{0}: \mathrm{d} t=-\sin \theta: \cos \theta: f,
\end{array}
$$

and $t_{\alpha}=t-\left(f z_{0} / \cos \theta\right)$ denotes the time at which the bicharacteristics through the point of consideration $x_{0}, z_{0}, t$, intersect with the plane $z=z_{0}=0$. According to equations (6) and $(7), \vec{v}_{1}^{(1)}$ is constant along the bicharacteristics generating characteristic surfaces $\xi=$ const. while $\vec{v}_{1}^{(2)} \cdot \vec{n}^{(1)}$ is a periodic function of $t$. Therefore,

$$
\int_{t_{a}}^{t}\left(\vec{v}_{1} \cdot \vec{n}^{(1)}+c_{1}\right) \mathrm{d} t=\frac{\gamma+1}{2} \vec{v}_{1}^{(1)} \cdot \vec{n}^{(1)} \frac{f}{\cos \theta} z_{0}+O(1) .
$$

Here the first term on the right side is unbounded as $z_{0} \rightarrow \infty$ and accounts for the cumulative effect of the wave amplitude associated with the velocity disturbances $\vec{v}_{1}^{(1)}$ on the distortion of characteristic surfaces in the $x, z, t$-space. Additional terms occur due to the mutual interaction of both wave families. However, since they are bounded, they are non-cumulative and can thus be neglected to leading order. Similar considerations hold for the second of equations (11) and, introducing the notation

$$
\vec{x}_{1}=\left(x_{1}, z_{1}\right),
$$

one therefore obtains

$$
\begin{aligned}
& \vec{x}_{1} \cdot \vec{n}^{(1)}=\frac{1}{2}(\gamma+1)(f / \cos \theta) \vec{v}_{1}^{(1)} \cdot \vec{n}^{(1)} z_{0}+O(1), \\
& \vec{x}_{1} \cdot \vec{n}^{(2)}=\frac{1}{2}(\gamma+1)(f / \cos \theta) \vec{v}_{1}^{(2)} \cdot \vec{n}^{(2)} z_{0}+O(1) .
\end{aligned}
$$


Evaluation of equations (15) and (16), taking into account equation (3), yields

$$
\begin{gathered}
x=x_{0}+\frac{1}{4}(\gamma+1)\left(f^{4} / k N^{2} \pi^{2}\right) z_{0} v_{x}\left(x_{0}, z_{0}, t\right)+O\left(\varepsilon^{2}\right), \\
z=z_{0}+\frac{1}{4}(\gamma+1)\left(f^{4} / k^{3}\right) z_{0} v_{z}\left(x_{0}, z_{0}, t\right)+O\left(\varepsilon^{2}\right),
\end{gathered}
$$

in full agreement with the results given by Nayfeh and Kelly [4] and Ginsberg [5].

Inspection of the second of equations (17) shows that $\left|\left(z-z_{0}\right) / z_{0}\right|=O(\varepsilon)$. Therefore $z_{0}$ may be replaced by $z$ in equations (15) and (16) to first order:

$$
\begin{aligned}
& \vec{x}_{1} \cdot \vec{n}^{(1)}=\frac{1}{2}(\gamma+1)(f / \cos \theta) \vec{v}_{1}^{(1)}\left(x_{0}, z_{0}, t\right) \cdot \vec{n}^{(1)} z+O(1), \\
& \vec{x}_{1} \cdot \vec{n}^{(2)}=\frac{1}{2}(\gamma+1)(f / \cos \theta) \vec{v}_{1}^{(2)}\left(x_{0}, z_{0}, t\right) \cdot \vec{n}^{(2)} z+O(1) .
\end{aligned}
$$

An alternative but to first order equivalent form of the expressions (17) is thus given by

$$
\begin{gathered}
x=x_{0}+\frac{1}{4}(\gamma+1)\left(f^{4} / N^{2} \pi^{2}\right) z v_{x}\left(x_{0}, z_{0}, t\right)+O\left(\varepsilon^{2}\right), \\
z=z_{0}+\frac{1}{4}(\gamma+1)\left(f^{4} / k^{3}\right) z v_{z}\left(x_{0}, z_{0}, t\right)+O\left(\varepsilon^{2}\right) .
\end{gathered}
$$

Equation (18) determines the component of $\vec{x}_{1}$ in the direction of $\vec{n}^{(1)}$ only. However, any amount of lateral straining will leave the structure of the wave motion associated with the characteristic surfaces $\xi=$ const. unchanged. Consequently, equations (18) and (19) describe the propagation of simple planar waves in the direction of $\vec{n}^{(1)}$ and $\vec{n}^{(2)}$, respectively. Furthermore, the complete wave pattern is obtained through linear superposition of both wave trains.

It should be noted that the above considerations lead to a generalization of results obtained by Kruskal and Zabusky [10], and by Mortell and Varley [11], who studied one-dimensional wave propagation phenomena in closed tubes. In this particular case the two wave families propagate in opposite directions $\left(\vec{n}^{(1)}=-\vec{n}^{(2)}\right)$ and the minor importance of wave interactions inside the gas column was first observed experimentally by Saenger and Hudson [12].

It is obvious that the considerations above are not restricted to the case of planar waves. Non-linear acoustic waves induced by a cylinder vibrating harmonically in a mode having a circumferential wave number have been calculated by Ginsberg [13] and by Nayfeh and Kelly [14], while non-linear waves induced by a sphere undergoing general harmonic pulsations have been investigated by Kelly and Nayfeh [15]. Again these solutions can be interpreted as the linear superposition of non-linear progressive wave trains which do not interact.

Department of Aerospace Engineering,

A. KLUwICK ${ }^{\dagger}$

University of Michigan,

Ann Arbor,

Michigan 48109, U.S.A.

(Received 12 May 1980)

\section{REFERENCES}

1. J. H. GINSBERG 1975 Journal of Sound and Vibration 40, 359-379. Multi-dimensional non-linear acoustic wave propagation, Part II: The non-linear interaction of an acoustic fluid and plate under harmonic excitation.

2. A. H. NAYFEH and A. KLUWICK 1976 Journal of Sound and Vibration 48, 293-299. A comparison of three perturbation methods for non-linear waves.

+On sabbatical leave from the Technical University of Vienna. 
3. A. H. NAYFEH 1977 Journal of Sound and Vibration 54, 605-609. Perturbation methods and non-linear hyperbolic waves.

4. A. H. NAYFEH and S. G. KELly 1978 Journal of Sound and Vibration 60, 371-377. Non-linear interactions of acoustic fields with plates under harmonic excitations.

5. J. H. GINSBERG 1978 Journal of Sound and Vibration 60, 449-458. A re-examination of the non-linear interaction between an acoustic fluid and a flat plate undergoing harmonic excitation.

6. J. H. GINSBERG 1979 Journal of Sound and Vibration 63, 151-154. A new viewpoint for the two-dimensional non-linear acoustic wave radiating from a harmonically vibrating flat plate.

7. E. LEITER 1971 in Übersichtsbeiträge zur Gasdynamik (editors E. Leiter and J. Zierep). Nichtlineare Ansbrietungs vorgänge. Wien, New York: Springer-Verlag. See pp. 13-94.

8. A. KLUWICK 1980 Progress in Aerospace Sciences (in press). The analytical method of characteristics.

9. A. KLUWICK 1974 Journal de Mécanique 13,131-157. Gleichmässig gültige Störtheorie und Kumulative Effekte bei Wellenausbreitungsvorgängen.

10. M. D. KRUSKAL and N. J. ZABUSKY 1964 Journal of Mathematics and Physics 5, 231-244. Stroboscopic perturbation procedure for treating a class of nonlinear wave equations.

11. M. P. MORTELL and E. VARLEY 1970 Proceedings of the Royal Society of London A 318, 169-196. Finite amplitude waves in bounded media: nonlinear free vibrations of an elastic panel.

12. R. A. SAENGER and G. E. Hudson 1960 Journal of the Acoustical Society of America 32, 961-970. Periodic shock waves in resonating columns.

13. J. H. GINSBERG 1978 Journal of the Acoustical Society of America 64, 1679-1687. Propagation of nonlinear acoustic waves induced by a vibrating cylinder. II. The three-dimensional case.

14. A. H. NAYFEH and S. G. Kelly 1979 Journal de Physique 40 Supplement, 8-13. Nonlinear propagation of waves induced by an infinite vibrating cylinder.

15. S. G. Kelly and A. H. NAYFEH 1981 (submitted to) Journal of Sound and Vibration. Non-linear propagation of general directional spherical waves.

\section{AUTHOR'S REPLY}

Kluwick's analysis [1] of the non-linear distortion of non-uniform planar waves by means of the method of characteristics represents an interesting alternative to the perturbation method of renormalization that had been employed earlier $[2,3]$ to study the same problem. Kluwick's work clearly demonstrates that the wave motion is a superposition of two sets of uniform planar waves. This author arrived at the same conclusion in a previous paper [4] by manipulating algebraically the perturbation solution. The superposition concept led there to enhanced understanding of the physics involved in the formation of shocks.

One basic disagreement between Kluwick's conclusions and those in reference [4] is whether the solution is a linear superposition. In Kluwick's notation, $x_{0}$ and $z_{0}$ denote strained co-ordinates corresponding to the Cartesian co-ordinates $x$ and $z$ parallel and perpendicular to the planar boundary. The straining functions for each are denoted as $x_{1}$ and $z_{1}$, and so the straining transformation has the form

$$
x=x_{0}+\varepsilon x_{1}+\cdots, \quad z=z_{0}+\varepsilon z_{1}+\cdots .
$$

Earnshaw's exact solution for a non-linear planar wave which is uniform perpendicular to its propagation direction $\vec{n}^{(i)}$ may be written, in the case of a small signal, as

$$
\vec{v}^{(i)}=\varepsilon \vec{n}^{(i)} g_{i}\left(t-f \beta^{(i)}\right)
$$

where $g_{i}($ ) denotes an arbitrary function, and

$$
\vec{n}^{(i)} \cdot\left(x \vec{e}_{x}+z \vec{e}_{z}\right)=\beta^{(i)}\left[1+\frac{1}{2}(\gamma+1) f \vec{v}^{(i)} \cdot \vec{n}^{(i)}\right]
$$

